\title{
LTE-Advanced Optimum Relay Placement with Zero Link Overflow Using AMMCS
}

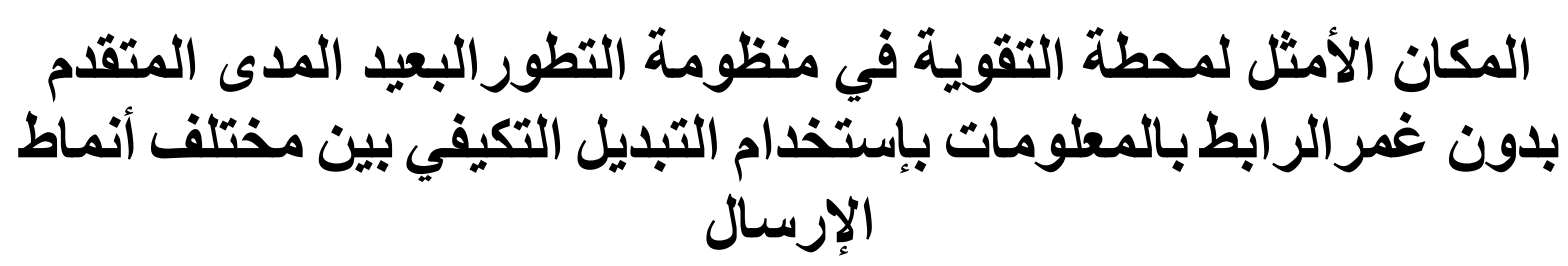

\author{
E.H. Abdelhay, F.W. Zaki, Sherif S. Kishk, H.S. Moustafa \\ Communication Engineering Dept., Faculty of Engineering, Mansoura
}

University, Egypt

تستخدم الثبكة متعددة المر احل باستخدام محطات التقوية لتوسيع التغطية وتعزيز كفاءة الطيف الترددي لتلبي

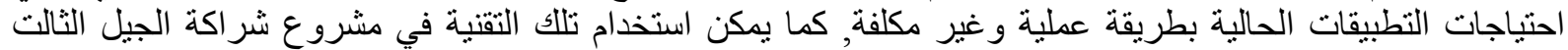

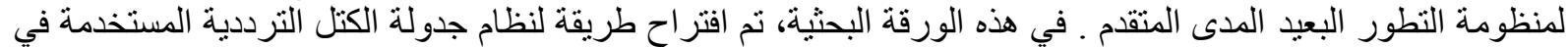

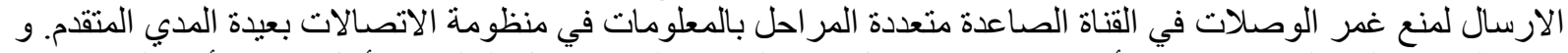

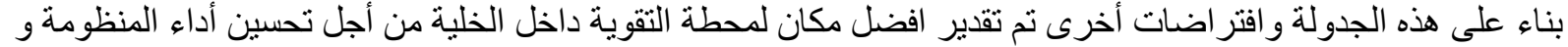
ذلك باستخدام معادلات المفاضلة الغير خطية. منوسط الإنتاجية، معدل الكسب في الإنتاجية ، ومتوسط زمن زمن نقل الملفات

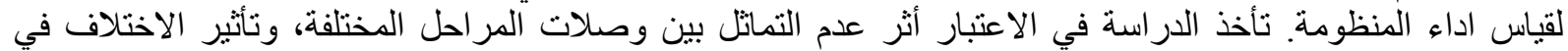

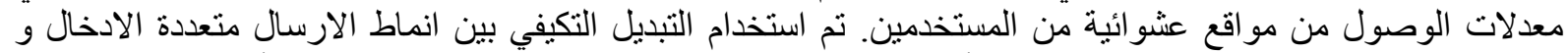

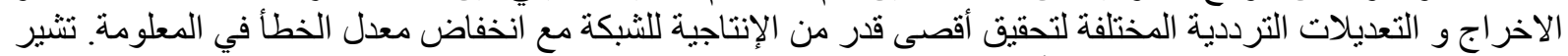

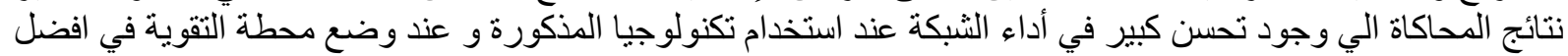
مكان تم تقديره في الخلية.

\section{Abstract}

Multi-hop Relay networks are proposed to fulfill the demanding coverage and capacity requirements for current applications in a cost efficient way. It can be used in IMT-Advanced technologies such as 3GPP LTEAdvanced. In this paper, Resource Blocks (RBs) proposed scheduling scheme is considered for zero multi-hop links overflow in Uplink LTE-Advanced. Then based on this scheduling scheme and other network assumptions the Optimum Relay Station (RS) placement in the cell was estimated using nonlinear optimization problem in order to improve LTE-A Uplink performance. The average throughput, Throughput gain, and Mean File Transfer Time (MFTT) are considered as performance measures. The study takes into consideration the impact of Asymmetry between multi-hop links, the effect of different arrival rates from random positions of UEs in the cell. Adaptive MIMO, Modulations, and Coding Switching (AMMCS) scheme is used to maximize network throughput with low bit error rate (BER). Simulation results show effective improvement in uplink network performance using the proposed scheduling scheme and AMMCS with RS at optimum location in the cell.

\section{Keywords:}

LTE-A, Multi-hop, Relay positioning, AMIMOS, AMC, Link overflow.

\section{Introduction}

LTE-Advanced offers better network performance than LTE Release 8 [1]. Most cellular networks research studies focus on downlink traffic scenarios, as most of wireless communications are a dominant traffic in downlink direction. However, in all $4 \mathrm{G}$ and all future networks this situation is changed, since popular file transfer and social network applications are supported. This requires large uplink traffic to solve the two links bottleneck problem. So this paper will focus on the LTE-A Uplink performance to overcome this problem.

Adding Relay station in the cell is an efficient LTE-A uplink Performance improvement technique. This technique 
helps Cell Edge Users (CEUs) to utilize Resource Blocks (RBs) usage. This will directly improve Cell Center Users (CCUs) performance and will improve total cell throughput, and maximum arrival rate [2].

LTE-A performance using relays have been considered in several studies. In [3],The RS placement and the bandwidth allocation were jointly optimized to maximize the downlink capacity for SISO relay networks. In [4], the optimal RS placement problem for coverage extension in downlinks SISO LTE-A was discussed. In [5] an adaptive MIMO detection algorithm for LTE-A system was proposed. In [6] LTE downlink switching scheme between multiplexing and diversity was discussed. There are few studies considered uplink performance in Multi-hop LTEnetworks. In [7] the authors considered SISO LTE uplink relay positioning to enhance cell throughput using RS neglecting the effect of RS-eNB link on throughput. In [8] the authors considered LTE-A uplink performance as a function of relay position using Adaptive MIMO switching and AMC techniques.

There are two main objectives for the present work. First, the proposed scheduling scheme named Optimum Time Fair Work Conservation (OTFWC) issued for zero link overflow and Uplink performance improvement. The second objective is focused to study the effect of adding Relay station (RS) on the uplink LTE-A performance, and to estimate the optimum position of RS in the cell based on Capacity Maximization Nonlinear Integer Problem (CMNIP). Solving this problem is proposed obtaining an approximate preliminary solution using exhaustive search. The metrics used to access the performance of the LTE-A network under consideration are: (1) Total Uplink Throughput: the total uplink data that can be received at eNB from all cell users per second using LTE-A uplink network parameters and assumptions. It can be defined as the total transmitted data from UEs to eNB in the all of simulation time divided by the total simulation time. (2) Mean File Transfer Time (MFTT): The mean time required for uploading average $1 \mathrm{Mbit}$ file from UEs located in the cell to the eNB. It's considered more helpful tool for RS and scheduling performance evaluation than throughput, as it refers to the time required for mean file transfer including the waiting time before transmission according to used scheduling scheme, and waiting list.

\section{Lte system features}

In this section, brief description of Physical RBs, UL Scheduling, and MIMO are presented.

\subsection{Resource Blocks}

In LTE-A the radio frequency spectrum is distributed for multiple users using SC-FDMA in uplink. The Resource Block ( $\mathrm{RB})$ is defined to consist of the intersection of 12 consecutive SC-FDMA sub-carriers with $180 \mathrm{kHz}$, each subcarrier has 7 SC-FDMA symbols with total time $0.5 \mathrm{~ms}$ for $10 \mathrm{MHz}$ bandwidth, 50RBs are provided. In addition a guard band of $1 \mathrm{MHz}$ is required [9].

\subsection{Lte Uplink Resource Scheduling Algorithms:}

LTE-A uplink scheduling have to utilize resource blocks (RBs) usage in such a way in order to maximize the spectral efficiency [10], Figure 1 illustrate the Fair Work Conserving (FWC) scheduling algorithm which provides better performance on average, this is due to the fact that it's RB scheduling is fair and channel unaware ( less delay than channel aware). 


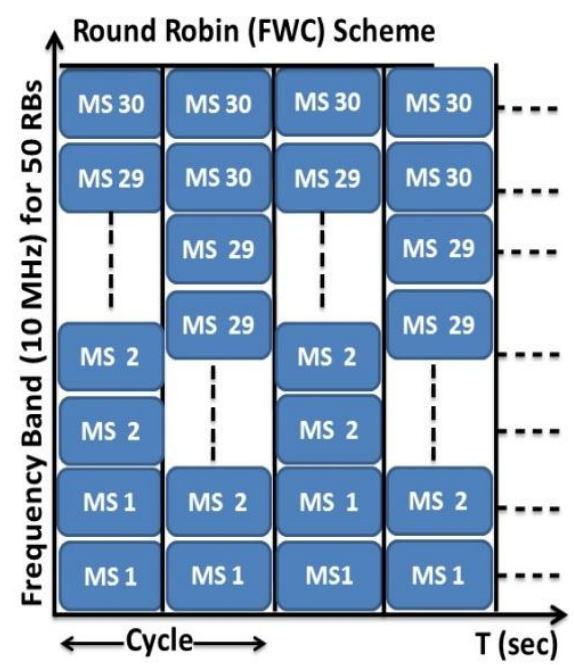

Figure 1. LTE-A UL FWC Scheduling

\subsubsection{FWC Scheduling using RS}

FWC Scheduling with RS is a little more complex than scheduling without RS.

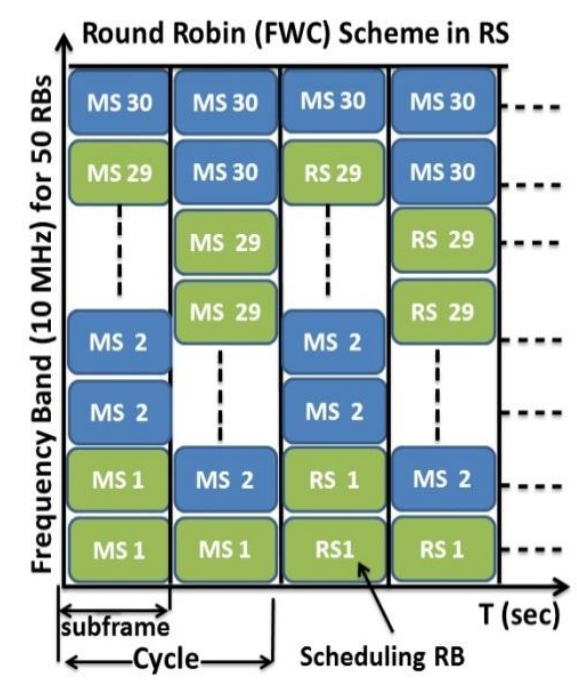

Figure 2.

Figure 2. FWC Scheduling with RS

To describe how the FWC in RS works, assume that for figure 2; Mobile stations (MSs) 1, and 29 are CEUs which have to use RS in transmission, in the first cycle MSs 1, and 29 use the scheduled RBs to transmit data to RS, and in the second cycle the RS resend these data to eNB. MSs 2, and 30 are CCUs who send to eNB directly in the two cycles. The disadvantage of this scheduling scheme is that some resources are wasted.

\subsection{Mimo Technique:}

AMC causes performance improvement in LTE system. MIMO can also improve network performance. There are different methods to make MIMO according to transmitting and receiving antennas configuration; as transmit diversity (TD), receive diversity (RD), and spatial multiplexing (SM). In transmit diversity the same information can be sent from multiple antennas using Multiple input single output method (MISO) [14]. In Receive diversity multiple antennas are used in receiving side using Single Input Multiple Output (SIMO) method. TD and RD improve SNR, and this affects the throughput indirectly. In Spatial Multiplexing the capacity can be increased using number of transmitting antennas which transmit independent data to increase transmitted data rate. SM can be achieved using beam forming which is supported by LTE-A networks [14].

\section{System Model}

This section presents the considered Channel capacity model, and link adaptation.

\subsection{Lte-A Uplink Channel Capacity Model}

The maximum data rate (throughput) that can be sent in a certain bandwidth can be obtained using Shannon's theory (1) for the maximum capacity of a communication channel [8].

$$
\mathrm{C}=\mathrm{B} \cdot \log _{2}(1+\mathrm{SNR})
$$

Where $\mathrm{C}$ is the maximum capacity in bits/second, B is the channel's bandwidth; SNR is the total received signal to noise power. In [12],[13] used different modulation and coding schemes to estimate the LTE-A spectral efficiency, and the results was lower than theoretical spectral efficiency, and from their results LTE-A uplink channel capacity model was: 


$$
\begin{aligned}
& \frac{\mathrm{R}_{\mathrm{b}}}{\mathrm{B}}= \\
& \begin{cases}\eta_{\mathrm{BW}} \cdot \log _{2}\left(1+\eta_{\mathrm{SNR}} \cdot \frac{\mathrm{s}}{\mathrm{N}}\right) ; & \text { for } \frac{\mathrm{s}}{\mathrm{N}} \leq \frac{\mathrm{s}}{\mathrm{N}_{\max }} \\
\frac{\mathrm{R}_{\mathrm{b}}}{\mathrm{B}_{\max }} & \text { for } \frac{\mathrm{s}}{\mathrm{N}}>\frac{\mathrm{s}}{\mathrm{N}_{\max }}\end{cases}
\end{aligned}
$$

Where $\eta_{\mathrm{BW}_{0} \mathrm{r}}$ and $\eta_{\mathrm{SNR}, \mathrm{r}}$ are bandwidth and SNR efficiencies respectively with values shown in table.1 $[12,13]$ as a function of number of transmitting antennas for uplink LTEAdvanced.

Table 1. SNR and BW efficiencies [12, 13]

\begin{tabular}{|c|c|c|}
\hline $\begin{array}{c}\text { Number of } \\
\text { trans. antennas }\end{array}$ & $\eta_{\mathrm{BW}, \mathrm{r}}$ & $\eta_{\mathrm{SNR}_{2} \mathrm{r}}$ \\
\hline 1 & $\mathbf{0 . 4}$ & $\mathbf{0 . 9 1}$ \\
\hline 2 & $\mathbf{0 . 3 8}$ & $\mathbf{1 . 0 5}$ \\
\hline 3 & $\mathbf{0 . 3 7}$ & $\mathbf{1 . 1 1}$ \\
\hline 4 & $\mathbf{0 . 3 4}$ & $\mathbf{1 . 2 4}$ \\
\hline
\end{tabular}

\subsection{Link Adaptation}

Adaptive Modulation and Coding (AMC) is Link adaptation technique which provides modulation scheme and coding rate adaptation. For data rate improvement higher order modulation schemes can be used providing more probability of error. To overcome this problem higher SNR can be used with high order Modulation schemes. Also coding rate can be chosen to be proportional to SNR. In [12], the authors generated the BER-SNR curves for the 13 different Modulation and Coding Schemes (MCSs) available in the LTE standard for $\mathrm{BER}=1 \mathrm{e}-3$ for Voice, video (live streaming), interactive gaming. In the LTE uplink AMC schemes are used according to CQI from 1 to 11 [13]. Adaptive MIMO is used in parallel with AMC switching to maximize the cell throughput and reduce MFTT with improved coverage [8]. AMMCS is an adaptive MIMO, modulation, and coding switching scheme with SINR thresholds listed in table 2 according to AMC-CQI list for BER 1e-3 with switching between two antenna configuration schemes (e.g., SD, and SM)
[8] to get better performance than standard 2x2MIMO.

Table 2. AMMCS switching thresholds

\begin{tabular}{|l|c|c|c|l|}
\hline CQI & $\begin{array}{c}\text { Max. } \\
\text { SNR }\end{array}$ & $\begin{array}{c}\text { Antenna } \\
\text { configuration }\end{array}$ & Modulation & $\begin{array}{l}\text { Code } \\
\text { rate }\end{array}$ \\
\hline 1 & -4 & 1x2SIMO & BPSK & 1 \\
\hline 2 & -2 & 1x2SIMO & QPSK & $1 / 3$ \\
\hline 3 & 0 & 1x2SIMO & QPSK & $1 / 2$ \\
\hline 4 & 2.1 & 1x2SIMO & QPSK & $2 / 3$ \\
\hline 5 & 3.8 & 1x2SIMO & QPSK & $4 / 5$ \\
\hline 6 & 6 & 1x2SIMO & 16QAM & $1 / 3$ \\
\hline 7 & 7 & 2x2MIMO & 16QAM & $1 / 3$ \\
\hline 8 & 7.8 & 2x2MIMO & 16QAM & $1 / 2$ \\
\hline 9 & 9.9 & 2x2MIMO & 16QAM & $2 / 3$ \\
\hline 10 & 12.6 & 2x2MIMO & 16QAM & $4 / 5$ \\
\hline 11 & 15 & 2x2MIMO & 16QAM & $5 / 6$ \\
\hline
\end{tabular}

\section{Performance Study}

A single LTE-A/E-UTRAN cell in urban area is considered with network parameters as given in table 3. Study is carried out for Uplink. The cell has one RS, whose position is variable and its best position in the cell is evaluated for higher Throughput, and lower MFTT, and results are compared with the optimum location resulted from the CMNIP. Cost-231hata path loss model is used [15], and [16]. Proposed OTFWC scheduling scheme is used in the RS, and results compared with the standard FWC scheduling scheme. New file transfers from random User Equipment (UEs) at random locations in the cell, are initiated with wide range of arrival rates $\lambda$ [16]. Adaptive MIMO-MC Switching (AMMCS) techniques are used in simulations [8].

Table 3. LTE-A Network parameters [2]

\begin{tabular}{|l|l|}
\hline Network Parameter & Value \\
\hline Cell radius & $1000 \mathrm{~m}$ \\
\hline $\begin{array}{l}\text { UE Maximum Trans. } \\
\text { Power }\end{array}$ & $23 \mathrm{dBm}$ \\
\hline Bandwidth of 1 PRB (W) & $180 \mathrm{KHz}$ \\
\hline Total bandwidth & $10 \mathrm{MHz}$ \\
\hline Number of RBs in10MHz & $50 \mathrm{RBs}$ \\
\hline Subcarrier spacing & $15 \mathrm{KHz}$ \\
\hline noise spectral density (No) & $-\mathbf{1 7 4 d B m} / \mathrm{Hz}$ \\
\hline Noise Figure of RSs (NF) & $5 \mathrm{~dB}$ \\
\hline Max SNR for uplink & $15 \mathrm{~dB}$ \\
\hline Carrier Frequency (Fc) & $\mathbf{2 . 6 ~ G H z}$ \\
\hline
\end{tabular}




\subsection{Cell Modeling}

The cell is divided into number of zones $(\mathrm{N})$ and number of sectors $(\mathrm{K})$ as shown in Figure 3. The intersection between each zone and sector named geometrical sector or segment, so the cell is divided into (N.K) segments. Each new UEs will be positioned on geometrical sector with position (i,j) where $(i=1,2, \ldots, N)$ and $(j=1,2, \ldots, K)$. Uniform random distribution for placing the UEs is considered. Calling the radius to zone $\mathrm{x}_{\mathrm{x}}$ so the zone radius can be calculated as follows:

$\int_{r_{x}}^{r_{x+1}} 2 \pi r \cdot d r=c$

With $r_{o}=0$ and $\mathrm{C}=$ surface per zone

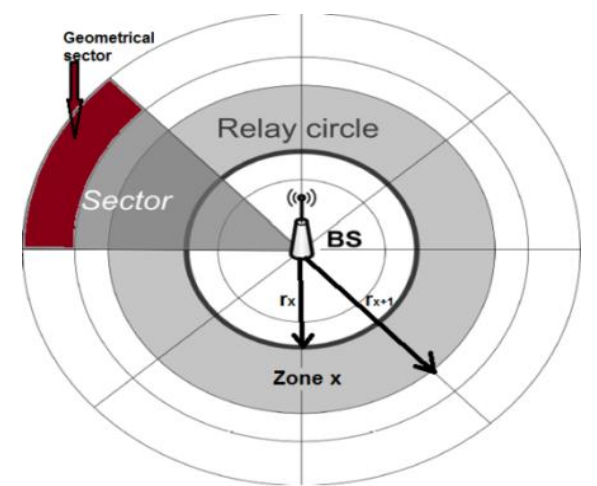

Figure 3. Cell with $\mathrm{N}$-zones and $\mathrm{K}$-sectors.

$\pi r_{x+1}^{2}-\pi r_{x}^{2}=C$

$r_{x+1}=\sqrt{\frac{C}{\pi}-r_{x}^{2}}$

Where $C=\pi r_{\text {cell }}^{2} / Z_{\text {total }}$

Where $r_{\text {cell }}$ is the cell radius and $Z_{\text {total }}$ is the total number of zones per cell.

\subsection{Estimating Distance between Ue And Rs}

As shown in Figure 4, the distance between UE and RS may be expressed as:

$d^{2}=r_{1}^{2}+r_{2}^{2}-2 \times r_{1} \times r_{2} \times \cos \phi$
Where: $r_{1}$ is the distance between UE and eNB, $r_{2}$ is the distance between RS and $\mathrm{eNB}$, and $\emptyset$ may be obtained as equation 10

$\emptyset=\frac{2 \pi}{K} \times \| j_{R S}-j_{U E} \mid$

Where $\mathrm{K}$ is the total number of sectors per cell, $j_{R S}$ the sector number where the relay station is placed, and $j_{U E}$ the sector number where the UE is placed.

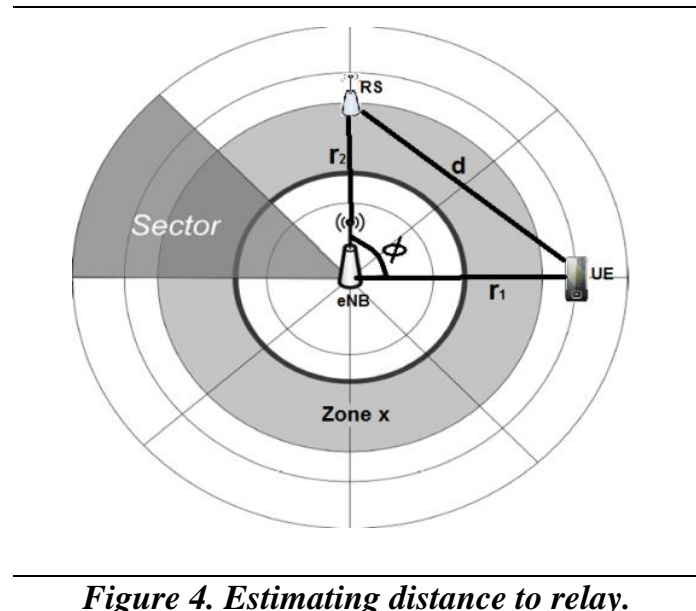

\subsection{Lte-Advanced Uplink Throughput Estimation}

The throughput of a UE in a physical segment at distance $\mathrm{x}$ from the (BS or RS) given $n_{t}$ transmitting antennas and $n_{r}$ receiving antennas is expressed as:

$$
\begin{aligned}
& \mathrm{C}(\operatorname{SNR}(\mathrm{x}))= \\
& \left\{\begin{array}{c}
0.4 \times R B \times W \times \\
\log _{2}(1+0.91 \times 2 \times S N R(x)) ; S N R \leq 7 d B \\
0.38 \times 2 \times R B \times W \times \\
\log _{2}\left(1+1.05 \times \frac{1}{2} \times S N R(x)\right) ; S N R>7 d B
\end{array}\right.
\end{aligned}
$$

Assuming perfect power control is used, so the SNR will be expressed as,

$$
\mathrm{SNR}(\mathrm{x})=\left\{\begin{array}{c}
\frac{\mathrm{P}_{\mathrm{T} \max }}{\mathrm{N}_{0} \times \mathrm{W}_{\mathrm{RRB} \times \mathrm{L}(\mathrm{x})}} ; \mathrm{SNR}<\mathrm{SNR}_{\max } \\
\mathrm{SNR}_{\max } ; \mathrm{SNR} \geq \mathrm{SNR}_{\max }
\end{array}\right.
$$

Where, $\mathrm{S} / \mathrm{N}(\max )$ is the $\mathrm{SNR}$ at which maximum LTE-A spectral efficiency is achieved RB is the number of used resource blocks per user according to used FWC 
scheme, path loss $\mathrm{L}(\mathrm{x}), \mathrm{W}$ is $180 \mathrm{kHz}$ bandwidth, No is the noise power spectral density. All UEs in the cell are assumed to have the same maximum transmit power $\mathrm{P}_{\mathrm{T}_{\text {max }} \text {, however: }}$

$\mathrm{P}_{\mathrm{T}_{\text {nnew }}}=\mathrm{SNR}_{\max } \cdot \mathrm{N}_{\mathrm{o}} \cdot \mathrm{RB} \cdot \mathrm{L}(\mathrm{x})$

Where

$P_{T_{-} \text {new }} \leq P_{T_{-} \max }$

\subsubsection{Throughput Estimation without relay}

The data rate of a UE in a segment at distance $\mathrm{r} 1$ from the $\mathrm{BS}$

$\mathrm{R}=\mathrm{C}(\mathrm{r} 1)$

Where the SNR can be estimated as:

$\operatorname{SNR}(r 1)=$

$\left\{\begin{array}{l}\frac{\mathrm{P}_{\mathrm{UE} \max }}{\mathrm{N}_{0} \times \mathrm{W} \times \mathrm{RB} \times \mathrm{L}(\mathrm{r} 1)}, S N R<S N R_{\text {max }} \\ S N R_{\text {max }}, S N R \geq S N R_{\text {max }}\end{array}\right.$

Where $\mathrm{P}_{\mathrm{UE} \_ \text {max }}$ is the maximum transmit power of the UE

\subsubsection{Throughput Estimation using relay for Asymmetric links with FWC}

Cell Center users (CCUs) are users who don't need relay station and transmit data to eNB directly with data rate higher than when using RS. The data rate of a $\mathrm{CCU}$ in a segment at distance $\mathrm{r} 1$ from the BS is estimated in equation 17.

$$
R(C C U)=C\left(r_{1}\right)
$$

Cell Edge users (CEUs) are users who need $\mathrm{RS}$ to transmit data to eNB with higher data rate than when transmit directly. The data rate of CEUs can be defined as the bottleneck of the UE-RS link and RS-eNB link as shown:

$$
R(C E U)=\frac{1}{2} \min \left[C(d), C\left(r_{2}\right)\right]
$$

The constant $(1 / 2)$ is placed in equation 18 as the half duplex mode RS is used. This is because the full duplex RS needs good beam forming to send and receive at the same time on the same RBs without interference, or dividing the available RBs between the two links, plus the delay effect in RS on the retransmission. In half duplex RS the CEUMS use the scheduled RBs to transmit data to $\mathrm{RS}$ for a cycle, and in another cycle the $\mathrm{RS}$ resend CEU's data to eNB. According to this the half of total data rate is achieved as shown in figure 5. The SNR of these two links have to be obtained as equations 19 , and 20 respectively.

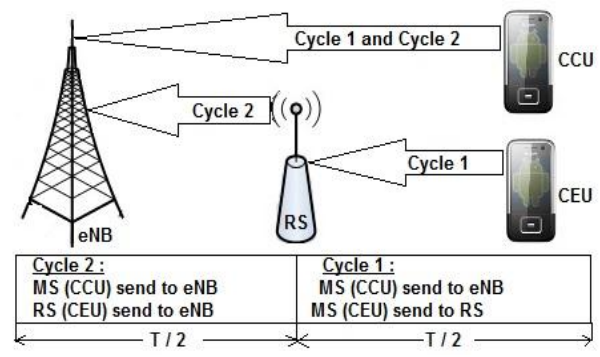

Figure 5. LTE-A UL transmission using RS

$\operatorname{SNR}(d)\left\{\begin{array}{c}\frac{\mathrm{P}_{\mathrm{UE} \max }}{\mathrm{N}_{\mathrm{o}} \times \mathrm{W} \times \mathrm{RB} \times \mathrm{L}(\mathrm{d})} ; S N R<S N R_{\max } \\ S N R_{\max } ; S N R \geq S N R_{\max }\end{array}\right.$

$\operatorname{SNR}\left(n_{2}\right)=$

$\left\{\begin{array}{l}\frac{\mathrm{P}_{\mathrm{RS} \max }}{\mathrm{N}_{\mathrm{o}} \times \mathrm{W} \times \mathrm{RB} \times \mathrm{L}(\mathrm{r} 2)} ; S N R<S N R_{\text {max }} \\ S N R_{\text {max }} ; S N R \geq S N R_{\max }\end{array}\right.$

\subsection{Otfwc Scheduling For Zero Link Overflow}

In order to utilize channel bandwidth efficiently, i.e., avoid wasting resource blocks and data overflow in multi-hop links, the amount of data transferred from MS to RS have to be equal the data transferred from RS to eNB. This can be done using modified FWC scheduling named Optimum Time Frame Work Conservation (OTFWC) with MS-RS transmission cycle time ( $\psi \cdot \mathrm{T})$, and RS-eNB transmission cycle time $((1-\psi) . \mathrm{T})$ as shown in fig.6, where ( $\psi . T)$ is inverse proportional to MS-RS link data rate, and $((1-\psi) . T)$ is inverse proportional to RS-eNB link. 
Using OTFWC equation 18 will be modified to be as follows:

$$
\begin{aligned}
& R(C E U)=\min \left[\psi C(d),(1-\psi) C\left(r_{2}\right)\right] \\
& \psi^{*} C(d)=\left(1-\psi^{*}\right) C\left(r_{2}\right)
\end{aligned}
$$

And then the optimum Time for the 1'st cycle will be:

$$
\psi^{*}=\frac{C\left(r_{2}\right)}{C(d)+C\left(r_{2}\right)}
$$

And according this the total CEU data rate using optimum time will be as follows:

$$
R(C E U)=\left[\frac{C(d) \times C\left(r_{2}\right)}{C(d)+C\left(r_{2}\right)}\right]
$$

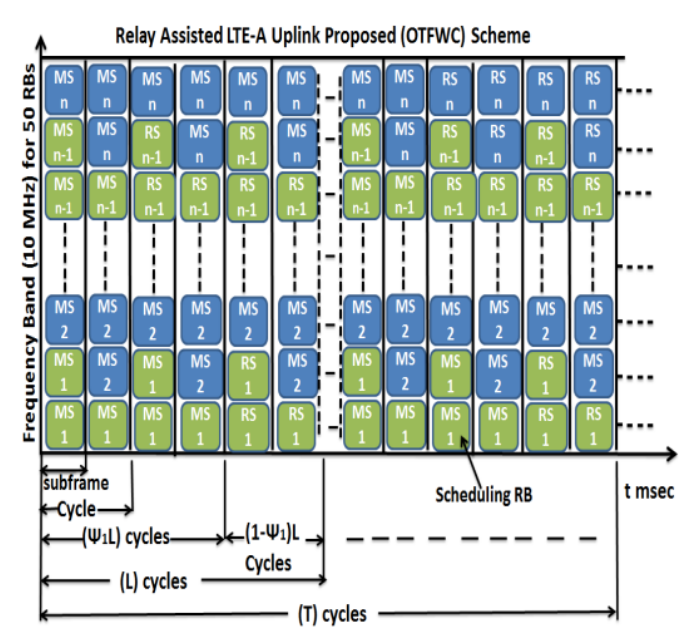

Figure 6. OTFWC transmission using RS

\subsection{Estimating Optimum Rs Placement}

The optimum location of the RS in the cell is calculated using the Capacity Maximization Nonlinear Integer Problem (CMNIP). It can be expressed as:

$$
\max _{\mathrm{X}, \mathrm{SNR}} \sum_{\substack{\mathrm{nEN} \\ \mathrm{meNU}_{\mathrm{H}}}} \mathrm{r}_{\mathrm{m}, \mathrm{n}}+\sum_{\mathrm{nEN}} \mathrm{N}_{\mathrm{CCUs}} \mathrm{r}_{\mathrm{n}}
$$

Subjected to:

$$
\begin{aligned}
& \sum_{\mathrm{m}=1}^{\mathrm{M}} \mathrm{X}_{\mathrm{m}}=1, \mathrm{~m} \in \boldsymbol{\mu} \\
& \mathrm{X}_{\mathrm{m}} \in\{0,1\} \\
& \mathrm{SNR} \leq \mathrm{SNR}_{\max }
\end{aligned}
$$

Equation (23) represents the objective function referred to the maximization of the total uplink throughput in the cell, with constraints expressed in equations 26, 27, and 28. Constraint (24) means that the total number of used RS in the cell is one RS. Constraint (25) means that the decision variable is binary $=0$ if the $R S$ is not at $C P$, and equals to 1 if the $\mathrm{RS}$ was at $\mathrm{CP}$. According to this fact, $\sum_{\mathrm{m}=1}^{\mathrm{M}} \mathrm{X}_{\mathrm{m}}$ means the total number of RSs assigned to $\mathrm{CP}$. Constraint (26) means that the received SNR cannot be more than the maximum acceptable SNR in the uplink LTE-A. This condition is achieved using power control algorithms.

Solving this problem is proposed obtaining an approximate preliminary solution using exhaustive search. The RS will be placed at the optimum CP in which maximum total uplink throughput is achieved.

\section{Simulation Setup}

First the cell is modeled with $\mathrm{N}$-zones and $\mathrm{K}$-sectors, $\mathrm{N}$ and $\mathrm{K}$ are chosen to large enough to establish a sharp site for each UE, RS, and the honor eNB. The performance measurements were done for each arrival rate $(\lambda)$ value from 1 to 18 with steps of $\lambda=0.25$. In the first part of simulation study, the number of zones $=\{1$, $2, \ldots, \mathrm{i}, \ldots, \mathrm{N}\}$ and sectors $=\{1,2, \ldots, \mathrm{j}$, $\ldots, \mathrm{K}$ \} used in this model are $\mathrm{N}=500$, and $\mathrm{K}=499$ respectively. 50 uniformly distributed UEs were assumed in the cell with distance r1 from the eNB, where $\mathrm{r} 1=\mathrm{U}(0,1000 \mathrm{~m}), \quad \varphi=\mathrm{U}(0,2 \pi), \quad$ where $\operatorname{rmax}=1000 \mathrm{~m} . \mathrm{M}=\{1,2, \ldots, \mathrm{m}, \ldots, \mathrm{M}\}$ is the candidate positions (CPs) for RS locations which are identified to be suitable for RS placement after site planning are. $\mathrm{M}$ assumed to be $31 \mathrm{CPs}$. The CPs begins from $25 \%$ of cell radius with step $1 \%$ of cell radius, and ending at $55 \%$ of cell radius. In this Preliminary section, the RS optimum location is estimated using exhaustive search. The RS will be placed at the optimum $\mathrm{CP}$ in which maximum total uplink throughput is achieved. 
In second step a loop is entered with one new iteration for each time step among all of simulation time. For each time step a random number of new UEs according to given arrival rate are assigned to random segment and added to queue. All UEs were classified whether there are cell edge users (CEU) or cell center users (CCU). According to given scheduling scheme (FWC, or OTFWC) each UE begins to transmit file directly to eNB if it's CCU or via $R S$ if it's CEU, when the file is completely transmitted; the UE is removed from the queue. At the end of simulation time the total cell throughput is obtained according to total transmitted files size and total required time for transmission, and from these results the maximum capacity and arrival rate will be obtained. The simulations were done for $2 \times 2 \mathrm{MIMO}$ with FWC scheduling, AMMCS with FWC scheduling, and AMMCS with OTFWC scheduling to compare performance. Finally, the previous simulations were repeated but with less number of zones and sectors, since many UEs need to arrive in a segment to obtain mean file transfer time for each segment. In this step as the simulation time is finished, the total upload time is divided by the total amount of complete files transmitted to obtain the mean file transfer time per segment, and then per cell. This step also was done using $2 \times 2 \mathrm{MIMO}$ with FWC scheduling, AMMCS with FWC scheduling, and AMMCS with OTFWC scheduling, to obtain the impact of different schemes on MFTT. The second step is repeated for different RS positions in the cell to compare the performance with the optimum RS location performance.

\section{Results}

Figs.7, 8, and 9 illustrate the LTEAdvanced total Uplink throughput for $2 \times 2 M I M O$ with FWC scheduling, AMMCS with FWC scheduling, and AMMCS with the proposed OTFWC scheduling; respectively. This is done using Cost231hata model without RS and with RS located at different positions.
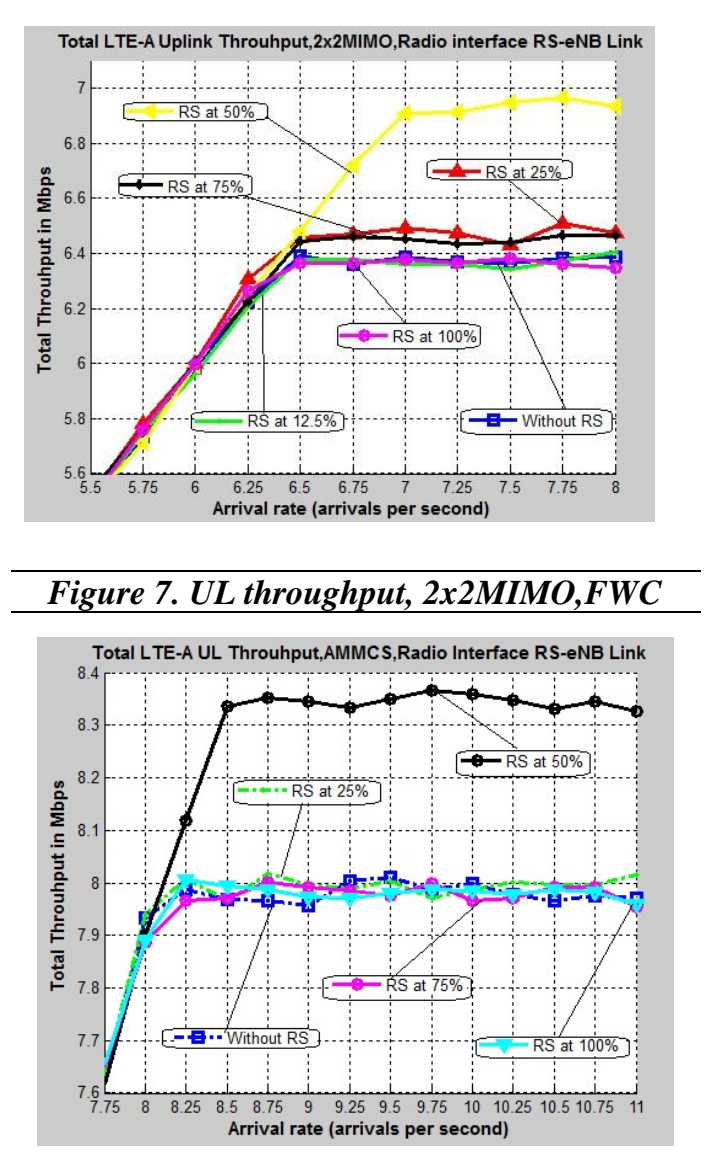

Figure 8. UL throughput, AMMCS, FWC

From these figures it can be observed that the maximum capacity was achieved with RS at $50 \%$ of cell radius. But it cannot be considered the best position for RS placement. However the throughput using $\mathrm{RS}$ at $50 \%$ of cell radius is increased by less than $10 \%$ of the throughput without using $\mathrm{RS}$. This may be considered as marginal increase since the total throughput is mainly affected by CCUs, beside the CEUs effect.

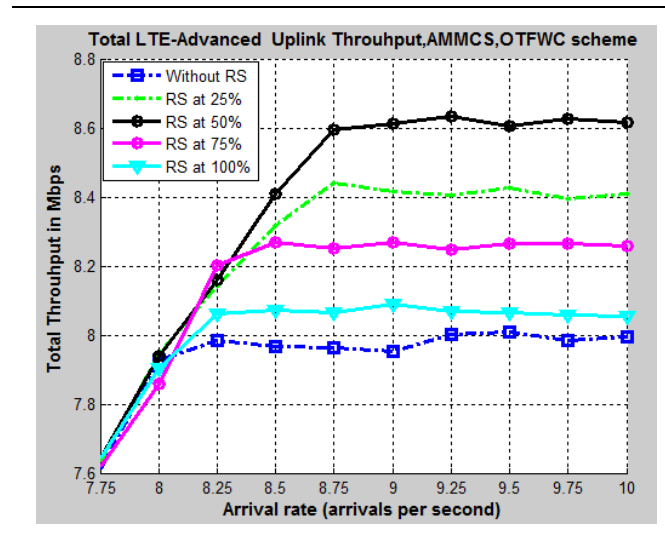

Fig 9. UL Throughput, AMMCS, OTF 
Moreover, it can be seen that the maximum throughput, Capacity, and arrival rate is achieved using AMMCS with proposed OTFWC scheduling schemes.

Another observation that can be noticed is: it does matter how fast the data rate per UE is as soon as the average arrival rate approaches maximum capacity. As the data rate per UE increases, when RS located in the best position in the cell, a MS finishes transmitting its file faster, and new users can be assisted at a faster rate. This is turn results in an increase in maximum capacity. Therefore, one can say that the maximum capacity will be increased with a higher average data rate.

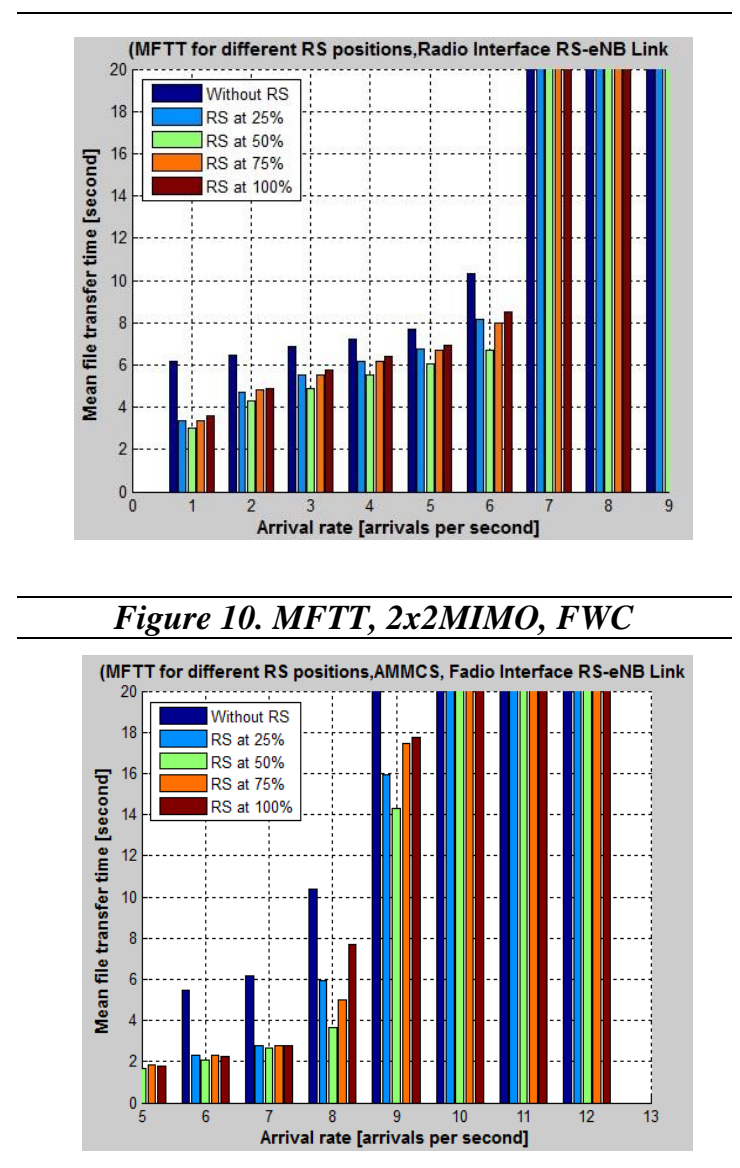

Figure 11. MFTT per cell, AMMCS, FWC

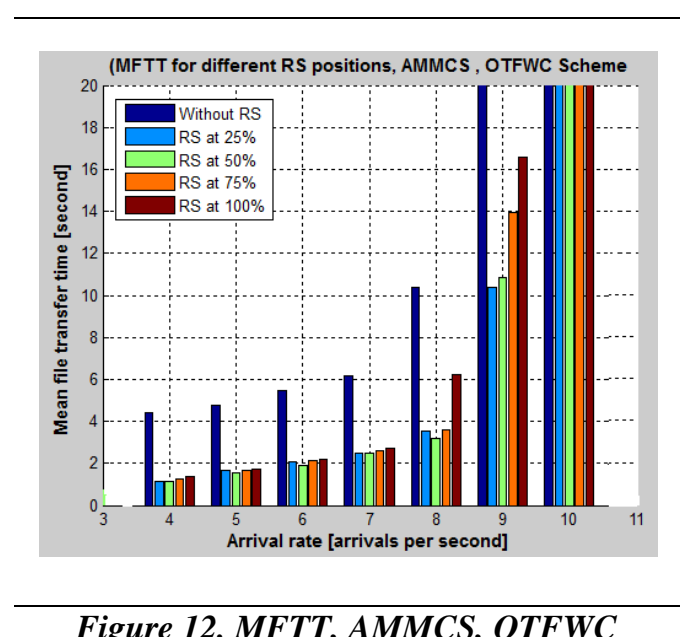

Figure 12. MFTT, AMMCS, OTFWC

Figs.10, 11, and 12 show the Mean file transfer time MFTT (sec per 1Mbit) for 2x2MIMO with FWC scheduling, AMMCS with FWC scheduling, and AMMCS with OTFWC scheduling; respectively. It can be seen from these figures that the MFTT for $\mathrm{RS}$ at $50 \%$ is always less than all other cases. Moreover, the lowest MFTT was achieved using AMMCS with OTFWC. The mean file transfer time goes infinite for $2 \times 2$ MIMO at arrival rate $(\Lambda)$ of 11 arrivals per second, for AMMCS with FWC at $\Lambda=12$ arrivals per second, for AMMCS with OTFWC at $\Lambda=12$ arrivals per second. However for AMMCS using OTFWC with $\Lambda=12$ arrivals per second, RS at 50\% don't saturate, whereas the MFTT schemes mentioned above reach saturation. So it can be concluded that AMMCS using OTFWC has the best performance according to MFTT.

The results conducted in figures 13, and 14 show the total uplink throughput, and MFTT; respectively for AMMCS with OTFWC scheduling using the optimum RS position resulted from the first step of simulations which be set to $37 \%$ of cell radius from the eNB. And these results are compared to the other fixed RS positions to show the optimum location gain. 

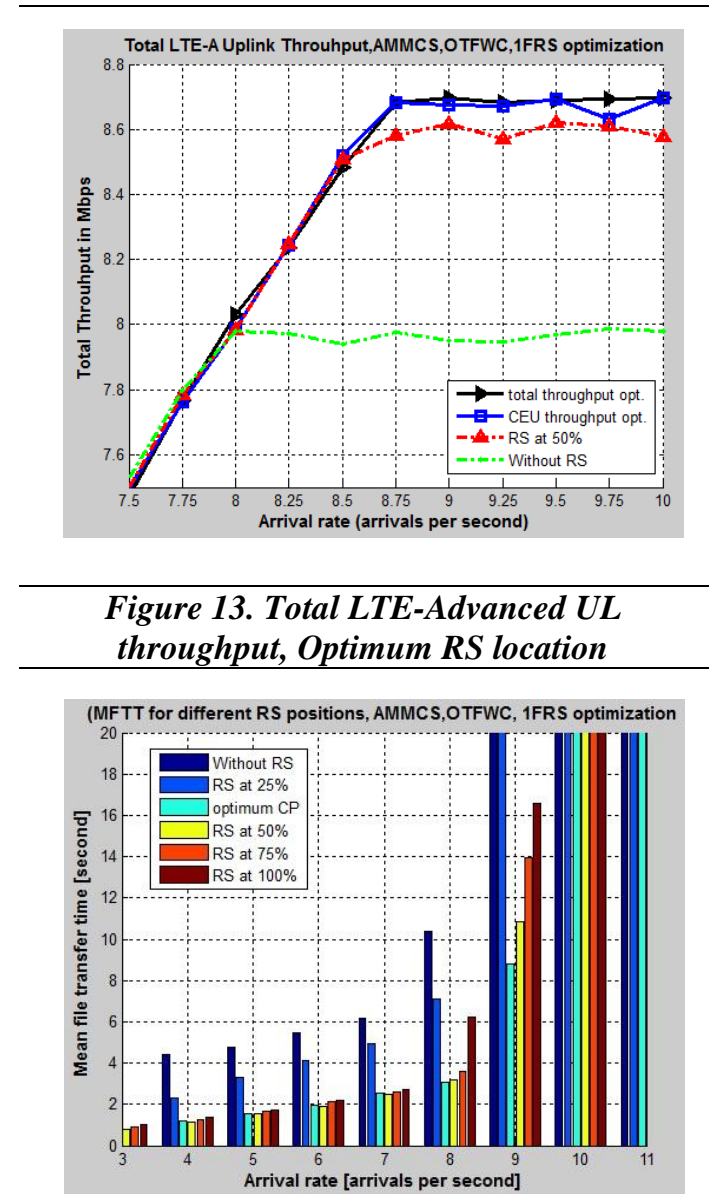

Figure 14. MFTT, Optimum RS location

From these figures it can be concluded that the RS at optimum location in the cell has the highest channel capacity, arrival rate, and lowest MFTT among all other locations. According to this fact, the $\mathrm{RS}$ at the optimum location improves the network performance significantly.

\section{ConClusions}

This paper has investigated the uplink performance improvements using Multihop Relay technology based on AMMCS in LTE-A networks. This research mainly answers two questions; where a relay station's would be positioned in the LTE-A cell for best performance, and how network performance is improved by eliminating the asymmetric multi-hop links data overflow using OTFWC scheduling scheme. In the present work, a model has been developed for an urban area single LTE-A cell.

The total LTE-A uplink throughput and system capacity was investigated as a function of arrival rates for the three scenarios. It was shown that under empirical path loss model like cost-231hata the RS placed at optimum location in the cell provides the highest total uplink throughput, highest system capacity, and lowest MFTT. Moreover, it can be concluded that OTFWC can overcome data overflow resulted from asymmetric links, so it can increase CEUs throughput resulting a significant increase in network capacity. Also it can be concluded that by using the AMMCS; CEUs achieve full use of available scheduled RBs. According to this; their throughput will be increased, and MFTT in the cell will be decreased. This due to the fact that, the AMMCS perform better than standard 2x2 MIMO in low SNR.

\section{REFERENCES}

[1.] A. Ghosh, R. Ratasuk, B. Mondal, and T. Thomas, "LTE-A: nextgeneration wireless broadband technology", IEEE Wireless Communications, vol. 17 , no. 3 , 2010, pp. 10-22.

[2.] E.H.Abdelhay, F.W.Zaki, S.S.Kishk, H.S.Mostfa, " Performance of Multihop LTE-A using AMC”, 32nd National Radio science Conference , pp. 104-114,2015.

[3.] B. Lin, P.-H. Ho, L.-L. Xie, X. Shen and J. Tapolcai, "Optimal RS Placement in Broadband Wireless Access Networks", IEEE Transactions on Mobile Computing, vol. 9, no. 2, 2010, pp. 259-269.

[4.] G. Joshi, "Optimal relay placement for cellular coverage extension", NCC, 2012.

[5.] X. Wu, L. Sun, M. Luo, "A Sphere Detection Based Adaptive MIMO Detection Algorithm for LTE-A", scrip Communications and Network journal, Vol.5, pp: 25-29, 2013.

[6.] M.U. Sheikh, R. Jagusz, J. Lempiäinen, "Performance Evaluation of Adaptive MIMO Switching in Long Term Evolution", 
7th International Wireless Communications and Mobile Computing Conference (IWCMC), 2011.

[7.] D.H. Hennepe, G. Karagiannis, "Impact of Relay Station Positioning on LTE Uplink Performance at Flow Level", Global Communications Conference, IEEE, 2012, pp.1586 1592.

[8.] E.H.Abdelhay, F.W.Zaki, S.S.Kishk, H.S.Mostfa, "Performance Evaluation of Adaptive MIMO-MC Switching in Uplink Multi-hop LTE-Advanced", Mediterranean Journal of Electronics and Communications (MEDJEC), Vol.11, No.1, 2015

[9.] M.A. Gadam, L. Maijama," A Review of Resourse Allocation Techniques for Throughput Maximization in Downlink LTE", Journal of Theoretical and Applied Information Technology, Vol. 58, No.2, pp.413-420, 2013.

[10.] D. Dimitrova, J. Berg, G. Heijenk, R. Litjens ,"LTE uplink scheduling Low level analysis", Master thesis, 1 University of Twente, Netherlands, 2 TNO ICT, Delft, The Netherlands, 2012.

[11.] K. Loa, C. Wu, S. Sheu, Y. Yuan, M. Chion, D. Huo, L.Xu, "IMTAdvanced Relay Standards", IEEE Communications Magazine, Vol. 48, No. 8, pp. 40-48, 2010.

[12.] P. Vieira, P. Queluz, A. Rodrigues, "LTE Spectral Efficiency using Spatial Multiplexing MIMO for Macro-cells" , 2nd International Conference on Signal Processing and Communication Systems (ICSPCS), 2008, pp1- 6.

[13.] M.U. Sheikh, R. Jagusz, J. Lempiäinen,"Performance Evaluation of Adaptive MIMO Switching in Long Term Evolution", 7th International Wireless Communications and Mobile Computing Conference (IWCMC), 2011.
[14.] H. Taoka, s. Nagata, K. Takeda, Y. Kakishima, X. She, and K. Kusame, " MIMO and COMP in LTEAdvanced, " NTT DOCOMO Technical Journal, Vol.12, No.2, pp:20-28, 2013.

[15.] S. Kale, A.N. Jadhav,"An Empirically Based Path Loss Models for LTE-A and Modeling for 4G Wireless Systems at $2.4 \mathrm{GHz}, 2.6 \mathrm{GHz}$ and 3.5 GHz," IJAIEM, Volume 2, Issue 9, pp.252-257, Sept, 2013.

[16.] E.H.Abdelhay, F.W.Zaki, S.S.Kishk, H.S.Moustafa, " Uplink Performance of Multihop LTE-A Based on Different Propagation Models", Mediterranean Journal of Electronics and Communications (MEDJEC), Vol.10, No.4, 2014, pp: 746

\section{Biographies}

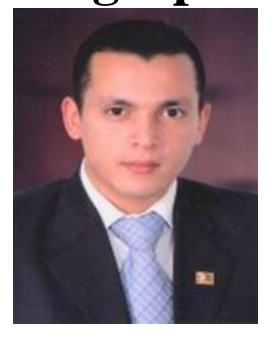

Ehab H. Abdelhay

Is born in Egypt 1983. He received the B.Sc. degree in Comm. Engineering from Mansoura University, Egypt in 2005. He received M.Sc. degree from the same university in 2010. He prepares for $\mathrm{PhD}$ degree in Electrical Communications Engineering. His research interest the area of Wireless Telecommunication Systems as $4 \mathrm{G}$, and $5 \mathrm{G}$ mobile systems, $\mathrm{He}$ is a Lecturer assistant at Faculty of Engineering, Mansoura University, Egypt. He worked there as a demonstrator from 2006, Lecture assistant from 2011.

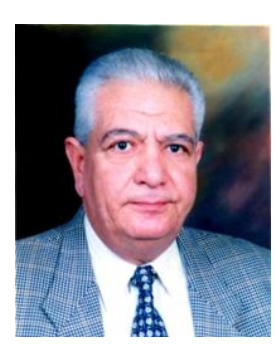

Fayez W. Zaki

Is born in Egypt 1957. He received B.Sc. from Communication Eng. from Menofia University Egypt 1969, M.Sc. Communication Eng from Helwan University

Egypt 1975, and Ph. D. from Liverpool University 1982. He is a professor at Faculty of Engineering, Mansoura University. He worked as a demonstrator at Mansoura University, Egypt from 1969, 
Lecture assistant from 1975, lecturer from 1982 , Associate Prof. from 1988, and Prof. from 1994. Head of Electronics and Communication Engineering Department Faculty of Engineering, Mansoura University from 2002 till 2005. Prof. Zaki supervised several MSc and PhD theses. He has published several papers in refereed journals and international conferences. $\mathrm{He}$ is now a member of the professorship promotion committee in Egypt.

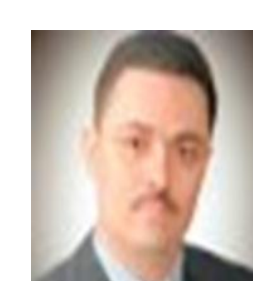

\section{Sherif S. Kishk}

Is an associate professor at Faculty of Engineering, Mansoura University. His experience covers, 3D object, watermarking,

Optical signal processing, Wireless networks, Circuits design, Biomedical image processing. He received the B.Sc. degree in Electronics and Communications
Engineering in 1992, Mansoura University, Egypt. He received M.Sc. degree in Computer Engineering in 2001 from the University of Connecticut, CT, USA. He received the Ph.D. degree in Computer Engineering in 2004 from the University of Connecticut, CT, USA. He has published several papers in refereed journals and conferences.

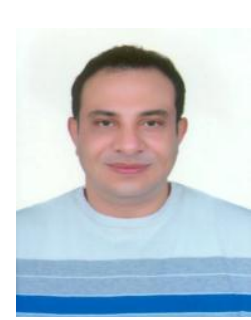

\section{Hossam S. Moustafa}

Is born in Egypt 1973. He received B.Sc. from Mansoura University Egypt in 2000. Ph.D degree from Mansoura University 2008. Fields of interest: Digital signal processing, Image processing, Tracking and Video Surveillance,

Biometrics, Mobile communications. He is an assistant professor at FEMU 